\title{
SOBRE EL APORTE DE LA FILOSOFÍA A LAS TEORÍAS DE CONCEPTOS EN CIENCIA COGNITIVA
}

\author{
Bernardo Aguilera \\ Universidad de Chile \\ bernardoad@u.uchile.cl \\ Bernardo Pino R. \\ Universidad de Las Américas \\ bpino@udla.cl
}

\begin{abstract}
RESUMEN / ABSTRACT
Este artículo defiende la relevancia de la filosofía en el estudio contemporáneo de conceptos. Con el desarrollo de la ciencia cognitiva, la teorización naturalista e interdisciplinaria acerca de los conceptos ha cobrado impulso. En este contexto, se ha argumentado recientemente que las teorías filosóficas de conceptos no son acerca del tipo de cosas que interesan a los psicólogos, descartando así la pertinencia científica de la contribución filosófica mencionada. Presentamos y discutimos dos casos que sugieren lo contrario, como un intento de reivindicar el papel crucial que tiene la filosofía en el desarrollo de teorías empíricas de conceptos.
\end{abstract}

Palabras clave: filosofía, ciencia cognitiva, teoría de conceptos, clases naturales.

ON THE CONTRIBUTION OF PHILOSOPHY TO THE THEORIES

OF CONCEPTS IN COGNITIVE SCIENCE

This paper defends the relevance of philosophy in the contemporary study of concepts. With the advent of cognitive science, naturalistic and interdisciplinary the orizing about concepts has gained momentum. In this context, it has been recently argued that philosophers' theories of concepts are not aimed at answering the issues that psychologists are interested in, thus dismissing the mentioned philosophical contribution as scientifically otiose. We present and discuss two cases in point suggesting otherwise, as an attempt to vindicate the crucial role of philosophy in the development of empirical theories of concepts.

KEYWORDS: philosophy, cognitive science, theories of concepts, natural kinds.

\section{Introducción}

\footnotetext{
R⿴囗十 Los conceptos son elementos teóricos fundamentales en la explicación científica de procesos cognitivos de orden superior, donde se considera que actúan como vehículos representacionales que posibilitan tales procesos. Si bien en sus orígenes el estudio de los conceptos fue eminentemente filosófico, durante las últimas décadas han proliferado teorías de conceptos desde el campo de la psicología experimental, instaurándose una investigación contemporánea sobre conceptos de carácter naturalista e interdisciplinar. En este escenario, el estudio de los conceptos se ha tornado complejo,y hoy en día suele asumirse que toda indagación sobre el tema debe estar empíricamente informada.
} 
Esta complejidad se ve reflejada en el desarrollo de múltiples teorías de conceptos, las cuales han pasado de ser recibidas con promisorio optimismo, a ser vistas como indicio de un estado de desorden y divergencia teórica en este ámbito de estudio. Un ejemplo paradigmático de esta última reacción es la propuesta de Machery (2009, 2010 y 2015), quien ha planteado que la teorización sobre conceptos ha dejado de aportar al progreso del conocimiento en ciencias cognitivas, y que éstas se beneficiarían con la eliminación de la noción de 'concepto' como elemento teórico unitario y transversal.

Las principales críticas a la propuesta eliminativista de Machery han defendido el estatus de los conceptos como clases naturales que participan en explicaciones de la psicología cognitiva, estatus que, como veremos en el próximo apartado, permite reivindicar su realidad independiente y objetiva. En el presente artículo elaboraremos una crítica distinta a la propuesta de Machery, esta vez dirigida hacia su escepticismo con respecto al aporte de la filosofía en la teorización contemporánea sobre conceptos.

En las tres primeras secciones de este trabajo establecemos el trasfondo teórico de la presente discusión, relativo a las teorías sobre conceptos en el marco de la ciencia cognitiva y el reciente debate respecto al estatus de los conceptos en tanto clases naturales. Luego, en la sección 4 presentamos la postura eliminativista de los conceptos propuesta por Machery, para luego evaluar de manera crítica su visión más general, acerca de la interacción entre filosofía y psicología en el estudio de conceptos. En las secciones 5 y 6 presentaremos dos ejemplos de teorización filosófica sobre conceptos que creemos permiten ilustrar la relevancia de la filosofía para el debate sobre conceptos en el marco de la ciencia cognitiva. El primero corresponde al análisis de la dimensión ontológica de los conceptos planteada por Vallejos (2008), mientras que el segundo ejemplo corresponde al trabajo de (Pino y Aguilera 2017), quienes problematizan acerca de la dimensión semántica de los conceptos en el contexto de la propuesta de Machery antes mencionada. Consideramos que estos ejemplos contribuyen a dar cuenta de la importancia de incorporar consideraciones filosóficas al momento de evaluar el grado de plausibilidad de las teorías de conceptos que surgen desde las actuales ciencias cognitivas, tema en el que profundizamos en nuestra discusión final.

\section{Psicología como ciencia y conceptos como clases naturales}

Bajo la influencia de Quine, en la filosofía de la psicología ha predominado la idea de que las teorías sobre conceptos no surgen tan solo de reflexiones a priori sobre nuestras intuiciones, sino que debieran estar informadas a partir de los hallazgos de la psicología empírica (Quine 1969, Thagard 2014). Esto se ha hecho manifiesto sobre todo a partir de los trabajos de la psicóloga cognitiva Eleanor Roch en los años 70, contribución que, a través del estudio de las capacidades de categorización de sujetos experimentales, motivó el surgimiento de teorías acerca de la estructura de los conceptos. Desde entonces, los conceptos han sido vistos como elementos de una teoría acerca de la cognición humana cuya naturaleza debe precisarse a través de los métodos que nos aporta la ciencia empírica, especialmente la psicología cognitiva (Carey 2009). 
Esta perspectiva descansa sobre ciertos supuestos metafísicos y epistemológicos que conviene establecer con claridad. Uno es el naturalismo, según el cual una descripción adecuada del mundo debe estar dada en términos de estados y procesos que ocurren dentro del orden natural. La mayor parte de los filósofos y psicólogos que estudian la mente suelen considerarse naturalistas en el sentido de situar a la mente como parte de este orden natural, en el cual, tal como señalan Botterill y Carruthers (1999), no es posible "admitir que exista algo en la mente que deba ser explicado invocando a espíritus vitales, almas incorpóreas, o cualquier otra cosa que no pueda ser integrada con la ciencia natural" (p. 1).

Es importante señalar que, si bien el naturalismo concibe al orden natural en términos fisicalistas, en el sentido de que cada evento físico posee una causa física que lo hace susceptible de ser capturado por explicaciones de la física fundamental, no presume de que la física sea la disciplina científica que deba explicarlo todo (Papineau 2009). Así, se ha hecho común la incorporación de disciplinas científicas distintas de la física -comúnmente llamadas 'ciencias especiales'- con sus propios dominios explicativos, que describen estados y procesos de un modo que no puede ser reducido a explicaciones de la física (Fodor 1974). Un caso paradigmático de ciencia especial es precisamente la psicología (Sterelny 1990; Crane 2001).

Otro supuesto metafísico importante es el realismo cientifico. Éste asume que la ciencia constituye la fuente de conocimiento más fidedigna que tenemos para informarnos sobre qué es lo que con mayor probabilidad hay en el mundo, y por tanto estaríamos epistémicamente justificados para asumir que los elementos teórico-explicativos de nuestras mejores teorías científicas designan clases de objetos (o propiedades) reales en el mundo, metafísicamente independientes de las mentes de los sujetos que las piensan (Fine 1999). Para el caso de los objetos mentales (e.g., conceptos, creencias, emociones) sería la psicología la ciencia encargada de determinar su estatus epistémico, vale decir, su probable existencia, al menos dentro del marco de nuestra visión científicamente informada de la realidad.

¿Qué hace que una cosa determinada forme parte de una teoría científica? Una visión popular a este respecto es la que los elementos teóricos de las teorías científicas deben reflejar las clases naturales presentes en el mundo. Las clases naturales corresponden a las categorías de cosas que la ciencia describe en su taxonomía para dar cuenta de la estructura metafísica del mundo. Más precisamente, una clase natural consiste en un conjunto de propiedades que se instancian en la realidad de manera homogénea y que tienen relevancia causal-explicativa para nuestras teorías científicas (Samuels y Ferreira 2010) ${ }^{1}$.

Tal como sucede en otras ciencias, el vocabulario teórico (exceptuando el lenguaje formal) de la psicología se refiere a clases naturales, viz., conjuntos de entidades que son objeto de las regularidades legaliformes descritas por la psicología. Sin embargo,

Aunque con una conclusión pesimista, Hacking (2007) proporciona una revisión panorámica de las principales tradiciones que han abordado el concepto de clase natural. 
a diferencia de otras disciplinas científicas, las clases naturales de la psicología no son identificadas con una propiedad física, sino que con su rol causal-funcional. En otras palabras, frente a la pregunta de qué es lo que está involucrado en poseer ciertas características mentales, la respuesta es que se trata de ciertos estados internos con tipos particulares de roles funcionales (Burwood et al. 1999). Por lo tanto, las teorías psicológicas se refieren a clases funcionales que, en principio, pueden ser realizadas en un número indefinido de sustratos físicos, toda vez que éstos ocupen los correspondientes roles causales (Sterelny 1990). Si bien estas clases funcionales son estados abstractos, permiten capturar generalizaciones explicativamente relevantes y por lo tanto pueden formar parte del inventario de las teorías científicas.

Para el caso de una teoría de conceptos, éstos corresponden a una parte sustantiva del vocabulario teórico de la psicología, siendo empleados para explicar una variedad de capacidades, tales como el aprendizaje, categorización, razonamiento, procesamiento lingüístico, etc. (e.g., Murphy 2002). Los conceptos pueden, por tanto, ser entendidos como clases naturales que la taxonomía psicológica distingue para construir sus predicados teóricos y formular sus generalizaciones empíricas, como, por ejemplo, que un episodio de pensamiento que contiene el concepto AGUA cause episodios de pensamiento que incluyen los conceptos LLUVIA o HUMEDAD, o que cause conductas relacionadas con ir en búsqueda de agua. En esta línea, los conceptos han sido tradicionalmente entendidos como una clase homogénea de objetos mentales, que participan de manera regular en procesos cognitivos que pueden subsumirse bajo leyes o generalizaciones legaliformes.

\section{Debate actual sobre conceptos: heterogeneidad y eliminativismo}

La psicología cognitiva ha visto proliferar líneas teóricas relativamente divergentes, siendo las principales aquellas que identifican los conceptos con prototipos, ejemplares, o bien teorías. Estas propuestas se erigen sobre el supuesto de que los conceptos son alguna clase de vehículos representacionales almacenados en la memoria que transportan información acerca de mundo (viz., sobre aquello que representan). Adicionalmente, los conceptos son entendidos como representaciones mentales estructuradas, vale decir, que poseen constituyentes que portan información relevante para el concepto. Un concepto es estructurado si sus partes se relacionan con otros conceptos, como por ejemplo, cuando se plantea que HUMEDAD y LLUVIA se relacionan con información constitutiva del concepto AGUA.

Este es el caso de las teoría que identifican los conceptos con prototipos², según la cual la estructura de un concepto corresponde a la representación de los rasgos (viz., información) más típicos de los objetos que caen en su extensión. En este sentido, la

2 Diversas contribuciones tempranas al surgimiento de la noción teórica de prototipos se pueden encontrar en Posner y Keele 1968, 1970; Rips, Shoben, y Smith 1973; Rosch 1973, 1975, 1978; Rosch y Mervis 1975; Rosch et al. 1976; Hampton 1979. 
caracterización más común que se hace de los conceptos en tanto prototipos corresponde a una representación sumaria de tales rasgos típicos (Prinz 2002). La teoría de prototipos ha sido pionera en plantear un enfoque psicológicamente realista para los conceptos, proporcionando explicaciones para fenómenos psicológicos tales como el efecto de tipicidad, i.e., la tendencia a categorizar instancias típicas más fácilmente que las atípicas.

Un conjunto alternativo de teorías, muy relacionado con la visión de prototipos, propone que los conceptos se pueden identificar con ejemplares ${ }^{3}$, en el sentido de que la estructura de un concepto corresponde a la representación de un conjunto de ejemplares de una misma categoría almacenados en la memoria. Por ejemplo, el concepto de ÁRBOL correspondería al conjunto (o una parte) de las representaciones formadas a partir de los árboles que un individuo ha encontrado a lo largo de su vida. Lo que sustenta esta última aseveración es la constatación empírica de que, mediante cálculos de similitud con respecto a instancias específicas de una categoría, los sujetos experimentales pueden realizar tareas de categorización más rápidamente que con respecto a representaciones prototípicas de las mismas categorías.

Un tercer modelo identifica los conceptos con una de dos ideas centrales: o bien los conceptos son teorías (e.g., Rips 1995), o bien son elementos de teorías (e.g., Gopnik y Meltzoff 1997). Estos enfoques caen bajo el rótulo de Teoría Teoría ${ }^{4}$ y surgen a partir de las dificultades que los modelos de prototipos y ejemplares tienen para dar cuenta de ciertos juicios de categorización más razonados, los cuales parecen manifestar un tipo de razonamiento que es característico de la teorización científica (Laurence y Margolis 1999). Ya que las teorías científicas van más allá de una mera descripción de los fenómenos que estudian, proporcionando también una explicación de los mismos, los investigadores que defienden este tercer grupo de teorías plantean que los conceptos corresponden a la representación mental de información que nos ayuda a explicar por qué ocurren las cosas. El modelo de teorías da cuenta de fenómenos psicológicos que difícilmente pueden ser explicados por los modelos de prototipos y ejemplares, como, por ejemplo, que el desarrollo conceptual pase por etapas de manera similar a las etapas por las que pasa el cambio de las teorías científicas (e.g., acumulación de contra-evidencia, ajustes teóricos, etc.), lo que ha llevado a algunos a plantear que el desarrollo cognitivo y la teorización científica son análogos y comparten mecanismos cognitivos (e.g., Gopnik 1996).

Cada uno de los tres modelos de conceptos mencionados más arriba (i.e., prototipos, ejemplares y teorías) cuentan con considerable respaldo experimental y existe cierto consenso en cuanto a sus fortalezas explicativas (Prinz 2002; Machery 2009). Sin embargo, cada uno de estos modelos atribuye distintas propiedades a los conceptos y por lo tanto no permiten consolidar una concepción unitaria y consistente

3 Véase Brooks (1978); Medin y Shaffer (1978); Nosofsky (1986), para algunos aportes paradigmáticos de estas teorías.

$4 \quad$ Contribuciones tempranas se encuentran en Murphy y Medin (1985); Carey (1985); Keil (1989). 
sobre qué son los conceptos. Individualmente, ninguno de estos modelos ha logrado dar cuenta satisfactoriamente de todas las propiedades que se espera que posean los conceptos, ni de todos los fenómenos que se espera que sean capaces de explicar. En consideración a esto último, se ha planteado que estaríamos frente a un escenario de desbarajuste, donde la investigación sobre conceptos discurre a través de supuestos teóricos y modelos difícilmente unificables en un marco teórico común (e.g., Smith y Samuelson 1997; Machery 2009 y 2010). Frente a este escenario, nos encontramos con tres posibles reacciones:

i. Prototipos, ejemplares y teorías nos entregan visiones distintas e incompatibles entre sí acerca de la naturaleza de los conceptos. Por lo tanto, son modelos que compiten entre sí para constituir la mejor explicación de la naturaleza de los conceptos y alguno de ellos debiera prevalecer en desmedro de los demás (e.g., Smith y Medin 1981; Murphy 2002).

ii. Prototipos, ejemplares y teorías nos entregan visiones distintas sobre la naturaleza de conceptos, pero son compatibles en el marco de algún modelo híbrido que caracteriza a los conceptos como un constructo teórico más heterogéneo de lo supuesto inicialmente (e.g., Laurence y Margolis 1999; Weiskopf 2009).

iii. Prototipos, ejemplares y teorías nos entregan visiones distintas e incompatibles entre sí acerca de la naturaleza de los conceptos. Sin embargo, cada uno de estos modelos tiene un sólido respaldo empírico que da cuenta de fenómenos cognitivos reales. Por lo tanto, es necesario rechazar la noción de concepto como clase natural unitaria. Esto tiene como resultado la eliminación de la noción de conceptos del vocabulario de la psicología.

Esta última reacción ha sido recientemente desarrollada por Machery (2009; véase también Piccinini y Scott 2006), generando gran controversia al proclamar frontalmente que "los conceptos debieran ser eliminados del vocabulario teórico de la psicología" (Machery 2009, p. 4).

\section{La eliminación de los conceptos y el cuestionamiento del rol de la filosofía}

Para comprender el argumento eliminativista de Machery, y lo que motiva nuestra discusión del mismo en el presente artículo, es importante tener presente que las clases naturales suelen ser entendidas como un conjunto de propiedades que se instancian en la realidad siguiendo los siguientes criterios (Boyd 1999; Samuels 2009; Griffiths 2004):

a. Son propiedades que tienden a presentarse de manera conjunta en la naturaleza en virtud de mecanismos causales que caen bajo principios teóricos comunes.

b. Tienen relevancia causal-explicativa para nuestras teorías científicas, en tanto contribuyen al razonamiento científico inductivo y contrafáctico. 
c. El descubrimiento de las propiedades y mecanismos específicos que subyacen a una clase natural son el producto de investigación científica, y por lo tanto son conocidos a posteriori (y no a priori o de manera puramente conceptual).

Ejemplos de clases naturales son elementos químicos tales como oro o agua, y especies biológicas tales como ballena o araucaria. En contraste, propiedades agrupadas de manera accidental no constituyen clases naturales; por ejemplo, objetos que miden dos metros de altura, prendas de vestir arrugadas, etc. Si determinada categoría científica corresponde o no a una clase natural puede ser, por tanto, materia de debate, el cual debe ser dilucidado en base a la evidencia empírica disponible. $\mathrm{Si}$, por ejemplo, determinada categoría científica resulta estar describiendo propiedades y mecanismos altamente heterogéneos (i.e., no satisface el criterio a), y en consecuencia no permite la realización de generalizaciones científicamente relevantes (i.e., no satisface el criterio b), su estatus como clase natural puede ser puesto en duda. Nótese que esto no implica necesariamente que aquella categoría deba ser eliminada ipso facto de alguna taxonomía científica, tal como lo ha ilustrado Cowie (2009) con respecto a la utilidad científica que han tenido ciertas concepciones erradas asociadas a los términos 'centro de gravedad', 'electrón' y, más extensamente discutido, 'gen'.

Siguiendo esta línea de razonamiento, Machery $(2005,2009)$ ha argumentado que, tras una revisión de la evidencia empírica disponible sobre conceptos, las teorías de conceptos describen una clase bastante heterogénea de mecanismos psicológicos, sin suficientes propiedades en común como para permitir la formulación de generalizaciones inductivas relevantes para una psicología científica. En consecuencia, sostiene que los conceptos no son una clase natural. Su propuesta positiva consiste en reemplazar la noción de concepto por la de distintas clases de representaciones llamadas 'cuerpos de conocimiento' (CdC), las cuales capturarían el rol de los mecanismos propuestos por los modelos de prototipos, ejemplares y teorías en procesos cognitivos de orden superior. De esta manera, por ejemplo, sería posible pensar sobre ballenas empleando tres tipos distintos de $\mathrm{CdC}$ : un prototipo de ballena, un ejemplar de ballena y una teoría de ballena. Estos tres tipos de representaciones mentales, a pesar de constituir mecanismos psicológicos distintos, serían correferenciales, viz., tendrían la propiedad de ser acerca de ballenas.

Un aspecto que consideramos particularmente provocador de la propuesta de Machery es que trasluce un marcado escepticismo respecto del rol de la filosofía en la teorización sobre conceptos. Después de todo, la idea de conceptos como fenómeno mental unitario proviene de una larga tradición en filosofía. Según Machery, filósofos y psicólogos persiguen objetivos diferentes al teorizar sobre conceptos, hasta el punto de no encontrarse teorizando sobre un mismo tipo de cosa. Su propuesta de abandonar la noción de concepto implica en gran medida desvincular a la filosofía de la teorización científica sobre conceptos, proponiendo que ésta siga su camino, con una terminología teórica propia, a través de la metodología experimental de la psicología cognitiva.

Si bien el autor reconoce que la noción de 'concepto' se emplea con distintos sentidos en psicología, considera que los psicólogos normalmente caracterizan los 
conceptos como "aquellos cuerpos de conocimiento que se encuentran almacenados en la memoria de largo plazo y que son utilizados en los procesos que subyacen a las competencias cognitivas de alto-orden" (Machery 2009 p. 10). Entonces, el propósito de la psicología sería caracterizar la naturaleza, formato, uso, adquisición y realización neuronal de estos cuerpos de conocimiento. Por otro lado, la filosofía de conceptos, de acuerdo con Machery, se ocupa de dilucidar "las propiedades en virtud de las cuales las personas pueden tener creencias, deseos -actitudes proposicionales en general-acerca de los objetos de esas actitudes. Una teoría [filosófica] de conceptos es, por lo tanto, una teoría semántica sobre nuestras actitudes proposicionales: se ocupa de explicar cómo nuestros pensamientos pueden tener el contenido que tienen" (op. cit. 2009, p. 33).

Este contraste entre filosofía y psicología respecto de la teorización sobre conceptos, puede describirse como una separación de tareas, donde "mientras los psicólogos explican la conducta y la cognición mediante la atribución de estados mentales con contenido, los filósofos explican cómo es que las personas tienen estados [mentales] con contenido" (op. cit., p. 31). Dicho de otro modo, de acuerdo con Machery, la filosofía se ocupa de temáticas de orden metafísico y semántico acerca de los conceptos, mientras que los psicólogos no tendrían que preocuparse de estos temas para poder desarrollar sus teorías acerca de las propiedades cognitivas de los conceptos.

Es importante notar que la afirmación de que psicólogos y filósofos tienen intereses distintos cuando teorizan sobre conceptos, no debiera tomarse como algo controversial. Muchos filósofos reconocen que es posible realizar progreso en el conocimiento acerca de procesos psicológicos que involucran conceptos (e.g., procesos de aprendizaje y categorización) sin necesidad de haber respondido preguntas filosóficas acerca de las condiciones de individuación o la naturaleza del contenido de los conceptos (Fodor 1994; Peacocke 1992; Rey 1998). Pero esto no quiere decir, por supuesto, que la indagación filosófica no tenga un carácter fundamental.

La postura de Machery, en cambio, es más bien escéptica de que las preguntas filosóficas sean relevantes para la teorización sobre conceptos. Por una parte, el autor sostiene que las estrategias de los filósofos para abordar cuestiones metafísicas sobre conceptos (e.g., las condiciones de posesión de un concepto, como las plantea Peacocke) presuponen que es posible obtener conocimiento a priori acerca de la naturaleza de los conceptos, lo cual resulta cuestionable dentro del marco filosófico naturalista propio de la ciencia cognitiva. Por otra parte, Machery considera que la metodología empleada por los filósofos para llevar a cabo su labor, típicamente intuiciones acerca de situaciones contrafácticas y otros ejercicios de análisis conceptual, además de estar poco desarrollada, puede ser puesta en duda a la luz de evidencia empírica reciente que demuestra la falibilidad de las intuiciones y los juicios de las personas. Y, como nos recuerda sarcásticamente Machery: "hasta donde yo sé, los filósofos son personas también" (2009 p. 46).

Aun cuando Machery (2010) reconoce que no es descartable que la filosofía progrese en desarrollar métodos más confiables para el estudio de los conceptos, su conclusión es categórica: "No obstante tanto filósofos como cientistas cognitivos [viz., psicólogos] usan el término 'concepto', no están hablando acerca de las mismas cosas 
[...] Muchas controversias entre filósofos y psicólogos acerca de la naturaleza de los conceptos son, por tanto, vacías" (p. 10). Para Machery, estas controversias no solo representan distintas posiciones en la investigación sobre conceptos, sino que además son una muestra de cómo la filosofía habría entorpecido el progreso de una psicología científica. En lo que sigue de este artículo, argumentaremos que la conclusión de Machery es demasiado apresurada y que, si bien es plausible plantear que existe cierta pluralidad semántica en el uso que filósofos y psicólogos dan al término 'concepto', y que asimismo es plausible que ambas disciplinas puedan hacer progresos de manera independiente, el objeto de estudio de filósofos y psicólogos que teorizan sobre conceptos sigue siendo la misma y las disquisiciones filosóficas en esta materia siguen siendo relevantes para una aproximación psicológica a los conceptos.

Nuestra estrategia consistirá en presentar dos ejemplos de teorización filosófica sobre conceptos, los cuales a nuestro juicio son exitosos en demostrar un diálogo fructífero entre filosofía y psicología en dicho ámbito de investigación. Nuestro primer ejemplo será la propuesta de Vallejos (2008) que apunta a los problemas que surgen en las teorías psicológicas sobre conceptos cuando descuidan la dimensión ontológica de los mismos. El segundo corresponde al trabajo de (Pino y Aguilera 2017), el cual nos permitirá ilustrar la relevancia del estudio de las propiedades semánticas en el marco del debate sobre teorías psicológicas de conceptos. Argumentaremos que, en ambos casos, filósofos y psicólogos tienen un mismo objeto de estudio y que es posible visualizar un trabajo colaborativo en el desarrollo de una teoría de conceptos sólidamente fundada y empíricamente informada.

\section{Aporte filosófico a la evaluación de teorías de conceptos en ciencia cognitiva}

Un aporte de la teorización filosófica a la investigación sobre conceptos se relaciona con la evaluación de teorías sobre conceptos en ciencia cognitiva. Un caso relevante en este sentido es la propuesta de Vallejos $(2002,2008)$, quien plantea que:

toda teoría experimentalmente basada contiene supuestos sustantivos acerca de la naturaleza de los conceptos que usualmente no son explicitados por los cientistas cognitivos y que no son cubiertos por la aplicación de los criterios pragmáticos de evaluación. El análisis ontológico debiera hacerse cargo de explicitar y de evaluar estos supuestos (2008, pp. 15-16).

Según Vallejos, el propósito de este análisis es determinar la naturaleza de los conceptos (i.e., aquello que son) por medio de la individualización de aquellas propiedades que son constitutivas de los mismos. En este contexto, Vallejos plantea una posición crítica frente al desarrollo de las teorías de conceptos que predominan dentro del marco de la psicología cognitiva y que hemos descrito en la sección 3 (i.e., las que proponen los modelos de prototipos, ejemplares, y teorías). Estas teorías, que llamaremos "teorías psicológicas de conceptos", a juicio de Vallejos (2008), suelen desestimar los aportes de la teorización filosófica dado el carácter a priori o trascendental de sus argumentos, 
los cuales no serían articulables con el marco empírico de evaluación de teorías que subyace a las teorías psicológicas de conceptos. Pero, a juicio del autor, la falta de fundamentos filosóficos que respalden los supuestos preliminares sustantivos acerca de la naturaleza de los conceptos, genera diversos problemas que no permiten que la elección de la mejor teoría psicológica se lleve a cabo de manera adecuada.

Vallejos expone con cierto detalle uno de estos problemas, relacionado con la evaluación entre teorías rivales dentro del campo de los conceptos. Toda teoría posee presupuestos ontológicos que determinan el alcance empírico de la teoría y el tipo de evidencia que permitiría confirmarla. Esto último puede manifestarse como un sesgo asociado a la manera en que alguna teoría en particular define los fenómenos que considera relevante explicar. Así, es posible que, tras un análisis ontológico enfocado en los supuestos que subyacen a ese tipo de sesgos, se demuestre que dos teorías rivales, que aparentemente explican los mismos fenómenos, realmente explican fenómenos distintos que no son comparables bajo criterios pragmáticos tales como el poder explicativo ${ }^{5}$. Dicho de otro modo, es posible que teorías que compiten por ser la mejor explicación no sean conmensurables entre sí, dada la existencia de diferencias sustantivas en su ontología. De ahí la importancia de tener claridad sobre los supuestos tanto metafísicos como metodológicos de las teorías al momento de evaluar cuál de ellas es la mejor de las disponibles en su campo.

En este sentido, la propuesta positiva de Vallejos (2008) es que los argumentos ontológicos debieran ser incorporados entre los criterios para juzgar qué teoría podría ser considerada como la mejor explicación. En palabras del autor:

[...] una evaluación que conduzca a establecer cuál es la mejor teoría de conceptos o qué forma tendría que asumir una teoría que satisfaga desiderata explicativos, no puede ser exhaustiva si depende solamente de argumentos metodológicos o de satisfacción de criterios pragmáticos generales. Estas instancias no son suficientes para proporcionar un apoyo evidencial adecuado para las presuposiciones sustantivas respecto de la naturaleza de los conceptos sobre los que se articula esa teoría (p. 44).

La incorporación de criterios o desiderata propiamente filosóficos permitiría entonces delimitar un territorio ontológico común sobre el cual comparar teorías rivales de conceptos $^{6}$. Por otra parte, motivar un análisis filosófico al momento de evaluar teorías obligaría a las teorías psicológicas de conceptos a hacer explícitos sus compromisos metafísicos, exponiéndolas a ser examinadas también en este aspecto. Tomemos por ejemplo las condiciones de individuación o identidad. Como vimos, las teorías psicológicas

Una teoría tiene mayor poder explicativo cuando abarca fenómenos que otras teorías del campo no explican y que sería deseable que explicaran.

6 Para autores que han propuesto desiderata filosóficos de este tipo, véase Fodor (1998), Prinz (2002), Laurence y Margolis (1999). 
de conceptos suelen operar bajo el supuesto de que los conceptos son representaciones estructuradas, en el sentido de que se individúan en función de las relaciones que sus partes constituyentes tienen con las partes constituyentes de otros conceptos. Vallejos (2002) acusa el riesgo de circularidad presente en este modo de individuación, por cuanto las condiciones de identidad de un concepto dependen de las de otro concepto, y así sucesivamente. El advertir oportunamente dificultades metafísicas de este tipo permitiría progresar de mejor manera y evitar el desarrollo empírico de teorías que carecen de una base metafísica que asegure su viabilidad.

En suma, Vallejos sostiene que la evaluación de teorías de conceptos basada únicamente en argumentos metodológicos y empíricos no puede aspirar a ser una evaluación exhaustiva. Por mucho que una teoría satisfaga criterios pragmáticos, tales como su adecuación a la evidencia experimental, no se puede inferir que dicha teoría posea una fundamentación ontológica consistente y comparable con las demás teorías con las que compite por dar la mejor explicación. En el mejor de los casos, solo se puede afirmar que una teoría así evaluada corresponde a la teoría que mejor satisface un consenso entre los investigadores del campo con respecto al tipo de cosas que se espera que una teoría pueda explicar. Vallejos admite que este consenso podría justificar la elección de la mejor teoría en el caso (ideal) de una ciencia de la mente consolidada; sin embargo, sostiene que esta situación aún no se puede vislumbrar, dada la multiplicidad de teorías, la diversidad metodológica y la consiguiente cantidad de presunciones sustantivas acerca de la naturaleza de los conceptos que esas teorías dejan sin explicar. De esta manera, dado el estado actual de desarreglo de las teorías de conceptos -e inmadurez de las ciencias cognitivas en general-, sería imprescindible incorporar el análisis ontológico como un antecedente más para la mejor explicación.

Es interesante notar que la propuesta de Machery que expusimos en la sección 4 puede ser vista como un trabajo de evaluación de teorías empíricas bajo un lente filosófico como el que promueve Vallejos. El diagnóstico de Machery es que el actual estado de desbarajuste que dificulta el progreso en la ciencia cognitiva de los conceptos se debería, al menos en parte, a un inadecuado uso del término 'concepto', el cual acabaría teniendo un referente con estatus ontológico tan dudoso que el autor propone erradicar el término del vocabulario teórico de la psicología. En este sentido, no deja de ser paradójico que Machery concluya que para favorecer el progreso de la investigación psicológica sobre los conceptos sea recomendable que la filosofía y la psicología sigan su camino por carriles independientes. Basta con señalar que tras eliminar el término 'concepto', Machery deja a la psicología operando sobre términos teóricos tales como 'información' o ‘cuerpo de conocimiento', cuyo estatus metafísico puede ser igualmente cuestionado. En cierto modo, la propuesta de arreglar las teorías de conceptos despachando el término 'concepto' junto con la filosofía nos parece tan poco razonable como, si se nos permite la comparación, el pretender disminuir la prevalencia de las enfermedades en un hospital a través de expulsar a los médicos que hacen los diagnósticos. 


\section{Aporte filosófico al problema de la correferencialidad de los CdC}

En el apartado 4 se describieron los rasgos más generales de la visión de Machery con respecto a los conceptos en psicología y su propuesta de incorporar la alternativa de diversos cuerpos de conocimiento correferenciales. Al asumir la correferencialidad entre estos $\mathrm{CdC}$, Machery presupone que existe alguna concepción viable del contenido de los mismos que pueda dar cuenta de esta correferencialidad. A continuación, nos centraremos en trabajo que hemos publicado en otra parte (Pino y Aguilera 2017), en el cual, a través de una discusión propiamente filosófica - en este caso de análisis del contenido semántico-, se cuestiona que los CdC propuestos por Machery sean compatibles con alguna teoría del contenido. Dado que gran parte del trabajo explicativo que hacen los conceptos en psicología consiste en dar cuenta de las propiedades semánticas del pensamiento, una propuesta alternativa sin una adecuada fundamentación filosófica acerca del contenido resulta, al menos, incompleta.

Para caracterizar las propiedades semánticas de los conceptos es posible distinguir, siguiendo grosso modo a Fodor y Pylyshyn (2015), dos tipos de enfoques. El primero de ellos, denominado 'Intensionalismo', plantea que el contenido de un concepto determina su extensión, mientras que, para el segundo enfoque, denominado 'Referencialismo', la extensión de un concepto determina su contenido. Puede decirse que las visiones más prominentes sobre la determinación del contenido conceptual caen bajo alguno de estos enfoques. Así, bajo el Intensionalismo se agrupan tanto aquellas que proponen que los conceptos obtienen su contenido por medio de sentidos fregeanos, como aquellas que plantean que dicho contenido está determinado por el rol inferencial de los conceptos. Para el caso del Referencialismo, el planteamiento básico es que el contenido de un concepto está determinado por algún tipo de relación causal y/o nomológica con su referente, siendo su versión más popular la Semántica Informacional. Sobre la base de estos dos enfoques, en lo que sigue evaluamos la posibilidad de que alguno de ellos pueda dar cuenta del contenido de los CdC propuestos por Machery.

Comencemos con los sentidos fregeanos. Diversos desarrollos filosóficos contemporáneos han seguido la distinción planteada por Frege entre sentido y referente, y la idea de que las expresiones de una lengua natural pueden poseer ambos, de tal manera que el sentido de una expresión contiene un 'modo de presentación' de su referente. Así, por ejemplo, 'ballena' y 'el mayor de los animales conocidos' pueden ser dos modos de presentación (o 'sentidos') de un mismo referente. Para Frege, asirse $e^{7}$ del sentido de una expresión es lo que posibilita acceder a su referente. Pero dado que los sentidos son objetos abstractos, autores neofregeanos (e.g., Dummet 1993) han tenido que postular condiciones de posesión de tales objetos con el fin de explicar la manera en la que podrían intervenir causalmente en nuestros procesos cognitivos.

Traducción que hacemos del término inglés "grasp", el que, a su vez, se utiliza como la traducción más común del término alemán "begreifen". 
Peacocke (1992) es quien mejor elabora estas ideas, planteando que un concepto en tanto sentido fregeano es individuable a través de las condiciones que un agente pensante debe satisfacer para poseer dicho concepto. Para Peacocke, estas condiciones corresponden a cierto conocimiento tácito acerca de las normas de individuación de un concepto, que son las que permiten realizar juicios en los que se aplica el determinado concepto. De acuerdo con esto, cuando el agente pensante satisface dichas condiciones de posesión de un concepto en tanto sentido, dicho concepto posee ipso facto su contenido. La pregunta que surge es si acaso una teoría como la de Peacocke puede adaptarse a los $\mathrm{CdC}$ de Machery, considerando ciertas semejanzas entre las condiciones de posesión de un concepto según Peacocke y los mecanismos epistémicos que dan cuenta de la posesión de CdC según Machery.

Para Machery, prototipos, ejemplares y teorías corresponden a distintos $\mathrm{CdC}$ distinguibles entre sí por el hecho de que cada uno es recuperado automáticamente de la memoria de largo plazo con el fin de ser utilizados en procesos cognitivos similares pero independientes entre sí, subyacentes a nuestras competencias cognitivas. Así, por ejemplo, lo que distinguiría a un prototipo-BALLENA de un ejemplar-BALLENA es que, mientras el primero es recuperado para participar en un mecanismo epistémico determinado (e.g., mecanismo de categorización basado en prototipos), el segundo lo sería para un mecanismo distinto (e.g., mecanismo de categorización basado en ejemplares). Si se acepta que estos mecanismos puedan ser entendidos como las normas de individuación de un concepto que los agentes pensantes deben satisfacer, entonces prototipos, ejemplares y teorías que son acerca de un mismo referente pueden ser entendidos, en el contexto de la visión de Machery, como tres modos de presentación distintos de ese referente. En este sentido, se podría afirmar que cuando utilizamos un $\mathrm{CdC}$ nos asimos de un sentido fregeano, ya que utilizar un $\mathrm{CdC}$ determinado implica la satisfacción de las condiciones de posesión de algún modo de presentación de cierto referente.

Aun dejando de lado el problema de explicar cómo objetos abstractos nomentales pudieran ser instanciados mediante mecanismos representacionales ${ }^{8}$, nuestra conclusión es que un enfoque neofregeano del contenido como el descrito no sirve para los propósitos de Machery. Para efectos de la presente discusión, basta con señalar que apelar a sentidos fregeanos para dar cuenta del contenido y la correferencialidad de $\mathrm{CdC}$ distintos no contribuye a la propuesta de desprenderse de la noción de conceptos, sino que más bien ratifica la necesidad de no hacerlo. La teoría de sentidos fregeanos asume que los agentes pensantes deben satisfacer condiciones que dan cuenta de la posesión de un concepto. Por lo tanto, si esas condiciones de posesión corresponden a mecanismos psicológicos adecuados para asirse de un sentido, entonces habría que decir que los correspondientes $\mathrm{CdC}$ tienen contenido conceptual. Con esto, se desvanecen

8 Para una respuesta a este problema en el marco de las ciencias cognitivas, véase Margolis y Laurence (2007). 
las motivaciones para cuestionar la existencia de los conceptos por el mero hecho de que diferentes $\mathrm{CdC}$ correferenciales dependen de mecanismos cognitivos distintos.

Como señalamos, una segunda visión dentro del Intensionalismo es aquella que sostiene que el contenido está determinado por el rol conceptual. En este caso, la idea central es que el contenido de un concepto está determinado por la relación que dicho concepto tiene con otros conceptos. Los modelos de conceptos descritos en el apartado 3 de este trabajo constituyen un caso paradigmático: prototipos y ejemplares asumen una relación estructural de similitud con otros conceptos, mientras que el modelo de teoría postula que el contenido de un concepto depende de sus relaciones inferenciales intrínsecas con otros conceptos dentro de alguna teoría adyacente. Dado que Machery fundamenta su propuesta de CdC en la evidencia empírica que sustenta la estructura inferencial de los modelos de conceptos antes mencionados, bien podría proponerse que los $\mathrm{CdC}$ heredan sus contenidos de los roles conceptuales de dichos conceptos. Sin embargo, lamentablemente, los $\mathrm{CdC}$ heredan también los problemas que tiene este enfoque intensionalista, siendo uno de ellos el carácter inestable del contenido. Dado lo variable de las relaciones inferenciales que se darían entre distintos $\mathrm{CdC}$, el contenido que surge de ellos no sería lo suficientemente constante para poder formular generalizaciones que permitan explicar fenómenos como el aprendizaje, el razonamiento y la comunicación interpersonal, entre otros.

Una posible salida a este problema es considerar la posibilidad de que un número limitado de inferencias pudiera asegurar un grado de estabilidad suficiente del contenido de una representación mental para dar cuenta, por lo menos, de la intuición básica de que es posible que pensemos acerca del mismo concepto en tiempos distintos, o que dos personas puedan compartir el significado de un concepto (o $\mathrm{CdC}$, si seguimos la terminología de Machery). Este es el caso de la versión de una semántica del rol conceptual que proponen Greenberg y Harman (2006), según la cual el contenido de una representación mental se deriva del uso que hacemos de estas representaciones en nuestra economía cognitiva. La visión de estos autores está comprometida con la idea de que la estabilidad del contenido está determinada por similitud, y no por identidad. Si esta teoría del contenido fuera aplicable a los CdC que propone Machery, podría explicar cómo diferentes $\mathrm{CdC}$ pueden tener contenidos estables y ser correferenciales por medio de un rol conceptual.

Sin embargo, un examen más detenido de la propuesta de Machery nos lleva a dudar de esta posible solución. Si la teoría del rol conceptual en cuestión funcionara para Machery, entonces el contenido de un CdC estaría determinado por el uso que hacemos de esa representación en nuestros procesos cognitivos. Según esto, por ejemplo, lo que un prototipo-ÁRBOL representa estaría determinado por la forma en que esa representación mental es usada para categorizar objetos del mundo que son árboles, y lo mismo aplicaría para un ejemplar-ÁRBOL y una teoría-ÁRBOL, mutatis mutandis. Pero aun cuando se conceda que la similitud de contenido entre instancias distintas de un mismo tipo de $\mathrm{CdC}$ puede ser suficiente para dar cuenta de la correferencialidad entre ellos (e.g., dos instancias de un prototipo-ÁRBOL), no se puede decir lo mismo con respecto a la correferencialidad de CdC diferentes (e.g., prototipo-ÁRBOL, ejemplar-ÁRBOL y teoría-ÁRBOL). La razón es que aun cuando distintos $\mathrm{CdC}$ acerca 
de un mismo objeto son usados en procesos cognitivos similares (e.g., categorización, inducción, pensamiento analógico, comprensión lingüística, etc.), cada uno es usado individualmente de manera muy diferente a los demás. Por ejemplo, tal como admite Machery (2010), mientras que los prototipos serían típicamente usados en procesos que computan similitud de manera lineal, los ejemplares son usados en procesos que computan similitud de manera no lineal, y, a su vez, las teorías serían normalmente usadas en procesos cognitivos similares a los algoritmos involucrados en el razonamiento causal. Por lo tanto, en la medida en que la similitud de contenido de los $\mathrm{CdC}$ derive de usos similares, lo esperable es que prototipos, ejemplares y teorías acerca de una misma categoría no compartan contenidos similares que garanticen su correferencialidad.

Podríamos extendernos en un estudio más detallado de la compatibilidad de los CdC con distintos enfoques del contenido, pero este no es el momento para tal análisis 9 . Para el propósito de esta sección, basta con destacar que un análisis de correferencialidad del contenido permite poner en duda la propuesta que Machery formula bajo el marco de teorías psicológicas de conceptos. A nuestro parecer, este aporte al debate del rol de los conceptos en psicología constituye otro ejemplo de cómo un examen filosófico, en este caso desde el ámbito de la teorización sobre el contenido semántico, es ineludible al momento de evaluar la plausibilidad de las teorías empíricas de conceptos o su reemplazo por constructos alternativos, tales como los CdC.

\section{Discusión y conclusiones}

Si bien es cierto que en sus orígenes el estudio de la mente en general, y de los conceptos en particular, fue eminentemente filosófico, también es cierto que el desarrollo en las ciencias de la cognición ha permitido progresos significativos en el estudio de la naturaleza y el funcionamiento de los conceptos. Para algunos autores, como Machery, estos avances podrían ser aún más notables si la filosofía fuese relegada a un rol periférico en la teorización sobre conceptos. De acuerdo con Machery, es incluso recomendable eliminar términos filosóficamente cargados, tales como 'concepto', y reemplazarlos por constructos teóricos basados en los hallazgos de la ciencia empírica. En este artículo hemos puesto en cuestión la propuesta de Machery y sus motivaciones para postular una división tan radical entre psicología y filosofía. Nuestra estrategia ha sido exponer dos ejemplos de contribuciones filosóficas que ponen de manifiesto el tipo de problemas que una teoría empírica de conceptos no debiera dejar de tener en consideración. La primera contribución se enfoca en las propiedades ontológicas de los conceptos y la segunda, en sus propiedades semánticas. De esta manera, hemos pretendido ilustrar lo relevante y fructífero que puede ser el aporte de la filosofía para el desarrollo y evaluación de teorías de conceptos en el marco de la ciencia cognitiva.

9 Para un análisis más detallado que sigue esta misma línea argumentativa, podemos remitir al lector a (Pino y Aguilera 2017). 
Evidentemente, existe una división de roles y metodologías entre filósofos y psicólogos al momento de teorizar sobre conceptos, pero los ejemplos antes descritos permiten visualizar ambos campos disciplinares como complementarios en torno a temas de interés común. Incluso creemos plausible sostener que, más que un mero complemento, existe una superposición de roles, tal como señalan Frankish y Ramsey (2012) en su introducción a un reciente volumen sobre ciencia cognitiva:

A decir verdad, la distinción entre ciencia cognitiva y la filosofía de la ciencia cognitiva no es tan nítida como uno pudiera suponer. Primero, realmente no hay una demarcación clara entre la investigación empírica, por un lado, y la reflexión filosófica sobre esa investigación, por el otro. Los cientistas cognitivos deben reflexionar acerca de las implicaciones generales de sus hallazgos, especular sobre materias más abstractas tales como supuestos ocultos y temas fundamentales, recurrir a experimentos mentales para argumentar sobre sus posturas, e invocar conceptos tradicionalmente filosóficos tales como conocimiento, representación y consciencia. Dicho de otro modo, hay mucho de razonamiento filosófico involucrado en ser un científico de punta (p. 2).

En concordancia con lo sostenido por estos autores, pensamos que la colaboración entre la teorización empírica y la filosófica es prácticamente imposible de desarticular, y es deseable que sea así. Nuestro planteamiento es que las teorías de conceptos constituyen un espacio especialmente propicio para este intercambio disciplinar, y que el proyecto separatista de Machery se origina a partir de una demarcación artificial entre filosofía y psicología de conceptos. Al mismo tiempo, es importante añadir que no basta con que los psicólogos se interesen por temas filosóficos, o que los filósofos tengan una orientación naturalista. Es también necesario que los filósofos se informen adecuadamente de los descubrimientos empíricos y la metodología propia de la investigación experimental, de manera que sus aportes sean relevantes y significativos para la psicología.

Las dos aproximaciones filosóficas al debate sobre conceptos en ciencia cognitiva que hemos expuesto en este artículo, viz., en relación con las propiedades ontológicas y semánticas de los conceptos, no agotan, por supuesto, los temas primariamente filosóficos que se han integrado a la teorización empírica sobre los conceptos, propiciando áreas de progreso genuinamente interdisciplinares. Otros ejemplos incluyen:

(a) Las propiedades sintácticas y semánticas de los conceptos, en particular la composicionalidad, la cual es entendida como la propiedad de una expresión compleja de estar determinada por el significado y la estructura de los conceptos que la componen. Estudiada originalmente por Frege, sigue siendo postulada como una condición de adecuación para las teorías de conceptos y sistemas representacionales en general (Hinzen et al. 2012).

(b) La relación entre pensamiento y lenguaje, debate filosófico acerca de la dirección de dependencia entre las propiedades semánticas y estructurales del pensamiento respecto de las del lenguaje con el que nos comunicamos. Mientras que algunos filósofos han planteado que la posesión de lenguaje es requisito para tener pensamiento conceptual (e.g., Dummett 1993), una 
postura contraria ha sido desarrollada desde la ciencia cognitiva a partir de los trabajos seminales de Chomsky en gramática generativa (Pinker 1994, Carruthers y Boucher 1988).

(c) Las propiedades normativas del pensamiento, en particular la racionalidad, rasgo que desde los comienzos de la filosofía se reconoce como un aspecto esencial del pensamiento humano. A partir de los trabajos de Kant, se ha planteado que la información que procesamos sobre el mundo adquiere un estatus propiamente conceptual cuando se configura dentro de un "espacio de razones" (McDowell 1994). La relación entre normatividad y pensamiento conceptual sigue siendo un debate vivo en ciencias cognitivas (e.g., Rey 2009).

(d) Las propiedades representacionales y computacionales de la mente, a partir de las cuales suelen formular supuestos filosóficos fundacionales de la ciencia cognitiva (Newell y Simon 1972). Visiones alternativas en ciencia cognitiva han intentado desafiar este modelo representacional-computacional de la mente, orbitando hacia posturas de carácter antirrepresentacionalista, inspiradas en la teoría de la percepción ecológica de Gibson $(1966)^{10}$. Este debate es claramente relevante para las teorías de conceptos, por cuanto las visiones alternativas recién mencionadas suelen prescindir de los conceptos entendidos como representaciones mentales.

Sin pretender ser exhaustiva, esta lista permite visualizar la variedad de temas donde resulta necesario el diálogo entre visiones de raigambre empírica de las ciencias cognitivas y la reflexión proveniente de la filosofía de la mente y la filosofía de la psicología. Si bien esto no garantiza, por sí solo, una solución al desarreglo que Machery alude con respecto al estudio actual de los conceptos, permite mostrar que el diálogo interdisciplinar es imprescindible si se quiere avanzar de manera fundamentada y consistente hacia una posible solución.

\section{Referencias bibliográficas}

Botterill, G., y P. Carruthers (1999), The philosophy of psychology. Cambridge: Cambridge University Press.

Boyd, R. (1999), "Homeostasis, species, and higher taxa”, en R. Wilson, ed., Species: New interdisciplinary essays. Cambridge: MIT Press; pp. 140-185.

10 Entre estas visiones alternativas destacan movimientos de fuerte base experimental, como el de las denominadas cognición situada y corporalizada (e.g., Brooks 1991; Clark 1997) y aquellos que promueven modelos de la cognición basados en sistemas dinámicos (e.g., Port y van Gelder 1995). 
Brooks, R. (1978), "Nonanalytic concept formation and memory for instances", en E. Rosch and B. C. Lloyd, eds., Cognition and concepts. Hillsdale, NJ: Erlbaum; pp. 169-211. 139-159.

(1991), "Intelligence without representation", Artificial Intelligence 47:

Burwood, S., Lennon, K. y P. Gilbert (1999), Philosophy of mind (Vol. 2). Montreal: McGill-Queen's Press.

Carey, S. E. (1985), Conceptual Change in Childhood, Cambridge, MA: MIT Press. (2009), The origin of concepts. Oxford: Oxford University Press.

Carruthers, P., y J. Boucher (1998), "Introduction: opening up options", en P. Carruthers y J. Boucher, eds., Language And Thought: Interdisciplinary Themes. Cambridge: Cambridge Univ Press; pp. 1-18.

Crane, T. (2001), Elements of mind. New York: Oxford University Press.

Clark, Andy (1997), Being there: putting brain, body and world back together again. Cambridge MA: MIT Press.

Cowie, F. (2009), “Why isn’t Stich an eliminativist?”, en D. Murphy y M. A. Bishop, eds., Stich and his critics. Hoboken: Wiley; pp. 14-74.

Dummett, M. (1993), The Seas of Language. Oxford: Clarendon Press.

Fine, A. (1998), "Scientific realism and antirealism", The Routledge Encyclopedia of Philosophy. Taylor and Francis. Recuperado de https://www-reproutledge-com.sheffield.idm.oclc.org/articles/thematic/scientific-realism-andantirealism/v-1/bibliography/scientific-realism-and-antirealism-bib.

Fodor, J. (1974), "Special sciences”, Synthese 28 (2): 97-115.

(1994), “Concepts: A potboiler", Cognition 50: 95-113.

(1998), Concepts: Where Cognitive Science Went Wrong, New York: Oxford University Press.

Fodor, J., y Z. Pylyshyn (2015), Minds without meanings: An essay on the content of concepts. Cambridge, MA: MIT Press.

Frankish, K. y W. Ramsey (2012), "Introduction”, en K. Frankish y W. Ramsey, eds., The Cambridge handbook of cognitive science. Cambridge: Cambridge University Press.

Gibson, J. J. (1966), The senses considered as perceptual systems. Boston: Houghton Mifflin.

Gonnerman, C. y J. Weinberg (2010), “Two Uneliminated Uses for 'Concepts': Hybrids and Guides for Inquiry", Behavioral and Brain Sciences 33: 211-212.

Gopnik, A. (1996), “The scientist as a child”, Philosophy of Science 63: 485-514.

Gopnik, A. y A. N. Meltzoff (1997), Words, thoughts, and theories. Cambridge, MA: MIT Press.

Greenberg, M., y G. Harman (2006), “Conceptual role semantics”, en E. LePore y B. Smith, eds., The Oxford handbook of philosophy of language. Oxford: Oxford University Press; pp. 295-322. 
Griffiths, P. (2004), "Emotions as Natural and Normative Kinds", Philosophy of Science 71(5): 901-911.

Hacking, I. (2007), "Natural Kinds: Rosy Dawn, Scholastic Twilight", Royal Institute of Philosophy Supplement 61: 203-239.

Hampton, J. A. (1979), "Polymorphous concepts in semantic memory", Journal of Verbal Learning and Verbal Behavior 18: 441-461.

Hinzen, W., M. Werning y E. Machery (2012), "Introduction”, en M. Werning, W. Hinzen, y E. Machery, eds., The Oxford handbook of compositionality. Oxford: Oxford University Press; pp. 1-16.

Keil, F. (1989), Concepts, Kinds, and Cognitive Development. Cambridge, MA: MIT Press.

Laurence, S., y Margolis, E. (1999), “Concepts and cognitive science”, en E. Margolis y S. Laurence, eds., Concepts: Core readings. Cambridge, MA: The MIT Press; pp. 3-81.

Machery, E. (2005), “Concepts are not a natural kind", Philosophy of Science 72: 444-467.

(2009), Doing Without Concepts. New York: Oxford University Press.

(2010), "Précis of Doing without Concepts", Behavioral and Brain Sciences 33: 195-244.

(2015), "By default", en E. Margolis y S. Laurence, eds., The Conceptual Mind. Cambridge, MA: The MIT Press; pp. 567-588.

McDowell, J. (1994), Mind and World. Harvard: Harvard University Press.

Margolis, E. y Laurence, S. (2007), “The Ontology of Concepts - Abstract Objects or Mental Re-presentations?”, Noûs 41(4): 561-593.

(2010), "Concepts and Theoretical Unification", Behavioral and Brain Sciences 33: 219-220.

Medin, D. L., y Schaffer, M. M. (1978), "Context theory of classification learning”, Psychological Review 85: 207-238.

Murphy, G. L., y Medin, D. L. (1985), "The role of theories in conceptual coherence", Psychological Review 92: 289-316.

Murphy, G. (2002), The big book of concepts. Cambridge: MIT Press.

Newell, A. y H. A. Simon (1972), Human problem solving. Englewood Cliffs, NJ: Prentice-Hall.

Nosofsky, R. M. (1986), “Attention, similarity, and the identification-categorization relationship", Journal of Experimental Psychology: Learning, Memory, and Cognition 115: 39-57.

Papineau, D. (2009), "Naturalism”, en E. N. Zalta, ed., The Stanford encyclopedia of philosophy. Recuperado de http://plato.stanford.edu/archives/spr2009/entries/ naturalism/.

Peacocke, C. (1992), A study of concepts. Cambridge, MA: MIT Press. 
Piccinini, G., y M. Scott (2006), “Splitting concepts”, Philosophy of Science 73: 390409.

Pinker, S. (1994), The Language Instinct. London: Penguin Books.

Pino \& Aguilera (2017), "Machery's Alternative to Concepts and the Problem of Content", Erkenntnis 1-21. https://doi.org/10.1007/s10670-017-9908-0

Port, R., y T. van Gelder (1995), Mind as motion: Explorations in the dynamics of cognition. Cambridge MA: MIT Press.

Posner, M. I., \& Keele, S. W. (1968), "On the genesis of abstract ideas", Journal of Experimental Psychology 77: 353-363.

Prinz, J. J. (2002), Furnishing the mind: Concepts and their perceptual basis. Cambridge, MA: MIT Press.

Quine, W. V. O. (1969), “Epistemology Naturalized”, en W. V. O. Quine, Ontological Relativity and Other Essays. New York: Columbia University Press; pp. 69-90.

Rey, G. (1998), “Concepts”, en E. Craig, ed., Routledge Encyclopaedia of Philosophy. London: Routledge. Recuperado de $<$ https://www.rep.routledge.com/articles/ thematic/concepts/v-1>.

(2009), "Resisting normativism in psychology", en BP McLaughlin y J. Cohen, eds., Contemporary debates in philosophy of mind. Oxford: Blackwell.

Rips, L. J., Shoben, E. J., \& Smith, E. E. (1973), "Semantic distance and the verification of semantic relations", Journal of Verbal Learning and Verbal Behavior 12: $1-20$.

Rips, L. J. (1995), “The current status of the research on concept combination", Mind y Language 10: 72-104.

Rosch, E. (1973), "On the internal structure of perceptual and semantic categories", en T. Moore, ed., Cognitive development and acquisition of language. New York: Academic Press; pp. 111-144.

(1975), "Cognitive representations of semantic categories", Journal of Experimental Psychology: General 104: 192-233.

(1978), "Principles of categorization", en E. Rosch y B. B. Lloyd, eds., Cognition and categorization. Hillsdale, NJ: Erlbaum; pp. 27-48.

Rosch, E. y C. B. Mervis (1975), "Family resemblance: Studies in the internal structure of catego-ries", Cognitive Psychology 7: 573-605.

Rosch, E., Mervis, C. B., Gray, W. D., D. Johnson, y P. Boyes-Braem (1976), “Basic objects in natural categories", Cognitive Psychology 8(3): 382-439.

Samuels, R. (2009), "Delusion as a natural kind”, en M. Broome, L. Bortolotti, eds., Psychiatry as Cognitive Neuroscience: Philosophical Perspectives (Chap. 3). New York: Oxford University Press; pp. 49-79.

Samuels, R. y M. Ferreira (2010), "Why dont concepts constitute a natural kind?" Behavioral and Brain Sciences 33 (2-3): 222-223. 
Smith, L. B., and L. K. Samuelson (1997), "Perceiving and remembering: Category stability, variability and development", Knowledge, concepts, and categories, K. Lamberts y D. Shanks, eds., Cambridge, MA: MIT Press; pp. 161-196.

Smith, E., \& Medin, D. (1981), Categories and Concepts, Cambridge, MA: Harvard University Press.

Sterelny, K. (1990), The representational theory of mind: An introduction. Oxford: Basil Blackwell.

Thagard, Paul, "Cognitive Science”, en E. N. Zalta, ed., The Stanford Encyclopedia of Philosophy (Fall 2014 Edition). URL $=<$ https://plato.stanford.edu/archives/ fall2014/entries/cognitive-science/>.

Vallejos, G. (2002), "Conceptos, representaciones y ciencia cognitiva" Revista de Filosofía 58: 145-170. Editores. (2008), Conceptos y ciencia cognitiva. Santiago: Bravo y Allende

Weiskopf, D. (2009), “The plurality of concepts”, Mind 169: 145-173. 